A. B. Kharazishvili, Institute of Applied Mathematics, University of Tbilisi, University str. 2, 380043 Tbilisi 43, Georgia

\title{
ON SOME SUBSETS OF SPACES EQUIPPED WITH TRANSFORMATION GROUPS
}

\begin{abstract}
For a given space $E$ equipped with a transformation group $G$, the notions of a $G$-thick set and of a $G$-thin set are introduced and discussed. Some relationships between these notions and the theory of $G$-invariant (more generally, $G$-quasiinvariant) measures are considered.
\end{abstract}

Let $E$ be a nonempty basic set and let $G$ be a subgroup of the group $\operatorname{Sym}(E)$ of all bijective mappings acting from $E$ onto $E$. In such a case the pair $(E, G)$ is usually called a space equipped with a transformation group.

We recall that a space $(E, G)$ is homogeneous if the group $G$ acts transitively in $E$, i.e. for any two points $x$ and $y$ of $E$, there exists a transformation $g$ from $G$ such that $g(x)=y$. It is well known that homogeneous spaces play an important role in various domains of modern mathematics.

We recall also that the group $G$ acts freely in $E$ if, for any two distinct transformations $g \in G$ and $h \in G$ and for each point $x \in E$, we have $g(x) \neq$ $h(x)$.

More generally, suppose that $I$ is a $G$-invariant $\sigma$-ideal of subsets of $E$. We say that $G$ acts $I$-freely in $E$ if, for any two distinct transformations $g$ and $h$ from $G$, the set $\{x \in E: g(x)=h(x)\}$ belongs to $I$.

Let $X$ be a subset of the basic set $E$.

Definition 1 We say that $X$ is $(G, I)$-thick (in $E$ ) if there exists a family $\left\{g_{k}: k<\omega\right\}$ of transformations from $G$ satisfying the relation

$$
E \backslash \cup\left\{g_{k}(X): k<\omega\right\} \in I,
$$

Key Words: transformation group, thick set, thin set, scattered set, countable equidecomposability, quasiinvariant measure, selector, nonmeasurable set.

Mathematical Reviews subject classification: Primary 28A05, Secondary 28A20.

Received by the editors August 27, 1996 
where $\omega$ denotes, as usual, the first infinite ordinal (cardinal) number.

Obviously, if $X$ is $(G, I)$-thick in $E$ and $X \subset Y \subset E$, then $Y$ also is $(G, I)$-thick in $E$.

Definition 2 We say that a set $X$ is $(G, I)$-thin (in $E$ ) if $X$ is not $(G, I)$-thick in $E$.

Clearly, if $X$ is $(G, I)$-thin and $Y \subset X$, then $Y$ also is $(G, I)$-thin.

Definition 3 We say that a set $X$ is $(G, I)$-scattered (in $E$ ) if, for any two distinct transformations $g$ and $h$ from $G$, the sets $g(X)$ and $h(X)$ are $I$-almost disjoint, i.e. $g(X) \cap h(X) \in I$.

Evidently, if $X$ is $(G, I)$-scattered and $Y \subset X$, then $Y$ is $(G, I)$-scattered, too.

Definition 4 We say that a set $X$ is a $(G, I)$-selector (in $E$ ) if there exists a selector $Y$ of the family of all $G$-orbits, such that $X \triangle Y \in I$, where $\triangle$ denotes, as usual, the operation of symmetric difference of sets.

If $I$ is a trivial $\sigma$-ideal, i.e. $I=\{\emptyset\}$, then each $(G, I)$-notion can be changed by the corresponding $G$-notion. In particular, a $(G,\{\emptyset\})$-thick set will be called a $G$-thick set, a $(G,\{\emptyset\})$-thin set will be called a $G$-thin set, etc.

Example 1. Let $\mu$ be a nonzero complete $\sigma$-finite measure defined on some $\sigma$-algebra of subsets of $E$. Let us denote by $I(\mu)$ the $\sigma$-ideal of all $\mu$-measure zero subsets of $E$. The measure $\mu$ is called $G$-quasiinvariant if the classes of sets $\operatorname{dom}(\mu)$ and $I(\mu)$ are invariant with respect to the group $G$. In this case, we can consider the notions of a $(G, I(\mu))$-thick set, of a $(G, I(\mu))$-thin set, of a $(G, I(\mu))$-scattered set, and of a $(G, I(\mu))$-selector.

Actually, we may restrict our further considerations to probability $G$ quasiinvariant measures on $E$, since every nonzero $\sigma$-finite measure is equivalent to a probability measure.

A particular case of the concept of a quasiinvariant measure is the concept of an invariant measure. We recall that a measure $\mu$ on $E$ is $G$-invariant if $\operatorname{dom}(\mu)$ is invariant with respect to $G$ and, for each set $Z \in \operatorname{dom}(\mu)$ and for each transformation $g \in G$, we have $\mu(g(Z))=\mu(Z)$.

Example 2. Let $(E, G)$ be a space equipped with a transformation group. It can easily be seen that

1) if $G$ is countable, then every $G$-selector is a $G$-thick subset of $E$; 
2) if $G$ acts freely in $E$, then every $G$-selector is a $G$-scattered subset of $E$;

3 ) if $G$ is uncountable and acts freely in $E$, then every $G$-selector is a $G$-thin subset of $E$.

In the present paper, we deal with some properties of $(G, I)$-thick, $(G, I)$ thin and $(G, I)$-scattered subsets of $E$, and discuss several applications of those properties to the theory of $G$-quasiinvariant ( $G$-invariant) measures.

We begin with the discussion of some relationships between $(H, I(\mu))$-thick sets and $(H, I(\mu))$-scattered sets, where $H$ is a countable group of transformations of $E$ and $\mu$ is an $H$-invariant measure on $E$. Let $X$ be an $(H, I(\mu))$-thick subset of $E$ and let $Y$ be an $(H, I(\mu))$-scattered subset of $E$. One can assert that if the sets $X$ and $Y$ are measurable with respect to $\mu$, then the inequality $\mu(Y) \leq \mu(X)$ is true. This result is essentially due to Minkowski who found various applications of it in the theory of convex sets and in geometric number theory (see, e.g., [4]). The proof of the Minkowski inequality is very simple. Indeed, we can write

$$
\mu(Y)=\mu(Y \cap E)=\mu(Y \cap(\cup\{h(X): h \in H\})) \leq \sum_{h \in H} \mu(Y \cap h(X)) .
$$

Taking into account the facts that $H$ is a group, $\mu$ is an $H$-invariant measure and $Y$ is an $(H, I(\mu))$-scattered set, we obtain

$$
\sum_{h \in H} \mu(Y \cap h(X))=\sum_{h \in H} \mu\left(h^{-1}(Y) \cap X\right) \leq \mu(X) .
$$

Thus, we have the desired inequality $\mu(Y) \leq \mu(X)$.

This simple result can immediately be applied to a question concerning the existence of a nonmeasurable set with respect to $\mu$ (in that case when $\mu$ is not identically equal to zero, of course). In order to show such an application, we need the following notion.

Definition 5 Let $(E, G)$ be a space equipped with a transformation group and let $\nu$ be a $G$-quasiinvariant measure on $E$. We say that $\nu$ is weakly metrically transitive if, for each $\varepsilon>0$, there exists a $(G, I(\nu))$-thick set $Z \in \operatorname{dom}(\nu)$ with $\nu(Z)<\varepsilon$.

This notion is closely connected with the usual notion of metrical transitivity of a quasiinvariant measure. We recall that a $G$-quasiinvariant measure $\nu$ on $E$ is metrically transitive (ergodic) if each set $Z \in \operatorname{dom}(\nu)$ with $\nu(Z)>0$ is $(G, I(\nu))$-thick in $E$. Let us observe that if $\nu$ is a nonatomic metrically 
transitive measure on $E$, then $\nu$ is weakly metrically transitive, too. The converse assertion is not true, in general. Moreover, it can easily be seen that every $G$-quasiinvariant extension of a weakly metrically transitive measure is also weakly metrically transitive, and it is well known that there are invariant extensions of the metrically transitive Lebesgue measure which are not metrically transitive. For instance, a nonseparable invariant extension of the Lebesgue measure, constructed by Kakutani and Oxtoby in their famous work [6], is not metrically transitive.

Proposition 1 Let $(E, G)$ be a space equipped with a transformation group, $H$ be a countable subgroup of $G$, and let $\mu$ be a nonzero $H$-invariant weakly metrically transitive measure on E. Suppose also that $H$ acts $I(\mu)$-freely in $E$. Then each $(H, I(\mu))$-selector is nonmeasurable with respect to $\mu$.

Proof. Let $Y$ be an arbitrary $(H, I(\mu))$-selector. Since $H$ acts $I(\mu)$-freely in $E$, the set $Y$ is $(H, I(\mu))$-scattered. Suppose, for a moment, that $Y \in$ $\operatorname{dom}(\mu)$. Let us fix $\varepsilon>0$. Since $\mu$ is weakly metrically transitive, there exists an $(H, I(\mu))$-thick set $X \in \operatorname{dom}(\mu)$ such that $\mu(X)<\varepsilon$. Applying the Minkowski inequality to the sets $X$ and $Y$, we get $\mu(Y)<\varepsilon$. But $\varepsilon$ was taken arbitrarily small, so we conclude that $\mu(Y)=0$. The latter equality immediately implies $\mu(E)=0$, because $Y$ is an $(H, I(\mu))$-thick set in $E$. Thus, we obtained a contradiction with the assumption that $\mu$ is a nonzero measure. This contradiction completes the proof.

Notice that the same argument simultaneously yields a more general result. Namely, each $(H, I(\mu))$-selector is nonmeasurable with respect to every $H$ invariant measure on $E$ extending the original measure $\mu$.

The following example shows that an analogue of Proposition 1 is not true for quasiinvariant measures.

Example 3. Let $H$ be an infinite countable group of transformations of $E$, acting freely in $E$. It is not hard to construct a probability weakly metrically transitive $H$-quasiinvariant measure $\nu$ on $E$ such that $\operatorname{dom}(\nu)$ coincides with the family of all subsets of $E$. In particular, all $(H, I(\nu))$-selectors are measurable with respect to $\nu$ and, hence, the statement analogous to Proposition 1 does not hold for $\nu$.

Of course, we can directly apply Proposition 1 to the classical Lebesgue measure given on a finite-dimensional Euclidean space (sphere) equipped with the group of all its isometric transformations. In this way we easily get a Lebesgue nonmeasurable subset of the space (sphere).

Here it is reasonable to remind that several approaches to the problem of existence of nonmeasurable sets are known. An algebraic approach is due to 
Vitali (1905), a topological approach is due to Bernstein (1908) and a purely set-theoretical approach was developed by Ulam (1930).

The first example of a Lebesgue nonmeasurable subset of the real line (equipped with the group of all its translations) was constructed by Vitali [17]. The standard Vitali argument can be found in various mathematical textbooks devoted to the theory of real functions, measure theory and related topics (see, e.g., a well-known book by Oxtoby [11] where Vitali, Bernstein and Ulam constructions are discussed in detail). Here we wish to notice only that the argument used in the Vitali construction, as well as the proof of Proposition 1 presented above, is based on the assumption of invariance of a given measure (cf. also [14] and [15] where some other constructions of nonmeasurable sets, with respect to nonzero $\sigma$-finite invariant measures, are considered). If we want to obtain the corresponding statement on the existence of nonmeasurable sets for nonzero $\sigma$-finite quasiinvariant measures, then we need an essentially different argument. We shall return to this question in our further considerations.

Now, we are going to show some natural relationships between $(G, I)$-thick sets and the theory of countable $(G, I)$-equidecomposability.

Let $(E, G)$ be a space equipped with a transformation group and let $X$ and $Y$ be any two subsets of $E$. We recall (cf., e.g., [18]) that $X$ and $Y$ are countably $(G, I)$-equidecomposable if there exist two disjoint families of sets

$$
\left\{X_{k}: k<\omega\right\},\left\{Y_{k}: k<\omega\right\}
$$

satisfying the following conditions:

a) for each index $k<\omega$, we have $X_{k} \subset X$ and $Y_{k} \subset Y$;

b) $X \backslash \cup\left\{X_{k}: k<\omega\right\} \in I$ and $Y \backslash \cup\left\{Y_{k}: k<\omega\right\} \in I$;

c) for each index $k<\omega$, the set $X_{k}$ is $G$-congruent with the set $Y_{k}$, i.e. there exists a transformation $g_{k} \in G$ such that $g_{k}\left(X_{k}\right)=Y_{k}$.

It is easy to see that if there exist two $I$-almost disjoint families

$$
\left\{X_{k}: k<\omega\right\},\left\{Y_{k}: k<\omega\right\}
$$

of sets satisfying conditions a), b) and c) mentioned above, then $X$ and $Y$ are countably $(G, I)$-equidecomposable.

Remark 1. Let $(E, G)$ be a space equipped with a transformation group and let $\mu$ be an arbitrary $\sigma$-finite $G$-invariant complete measure on $E$. Then the following assertions are equivalent: 
1) $\mu$ is metrically transitive;

2) for any two $\mu$-measurable sets $X$ and $Y$ such that $\mu(X) \leq \mu(Y)$, the set $X$ is countably $(G, I(\mu))$-equidecomposable with a subset of $Y$.

This result is essentially due to Banach and Tarski (see [2]).

Also, if $\nu$ is an arbitrary $\sigma$-finite $G$-quasiinvariant complete measure on $E$, then the following assertions are equivalent:

(1) $\nu$ is metrically transitive;

(2) for any two $\nu$-measurable sets $X$ and $Y$, either $X$ is countably $(G, I(\nu))$ equidecomposable with a subset of $Y$ or $Y$ is countably $(G, I(\nu))$-equidecomposable with a subset of $X$.

Thus, we see that the metrical transitivity of a given $\sigma$-finite invariant (quasiinvariant) complete measure can be expressed in terms of countable equidecomposability, modulo the $\sigma$-ideal of measure zero sets.

Lemma 1 Let $(E, G)$ be a space equipped with a transformation group, I be a $G$-invariant $\sigma$-ideal of subsets of $E$, and let $X$ be a subset of $E$. Then the next two assertions are equivalent:

1) $X$ is a $(G, I)$-thick subset of $E$;

2) $X$ contains an $(H, I)$-selector, for some countable subgroup $H$ of $G$.

Proof. Suppose that the set $X$ is $(G, I)$-thick in $E$. Then there exists a family $\left\{h_{k}: k<\omega\right\}$ of transformations from $G$ such that

$$
E \backslash \cup\left\{h_{k}(X): k<\omega\right\} \in I .
$$

Denote by $H$ the subgroup of $G$ generated by $\left\{h_{k}: k<\omega\right\}$ and put

$$
Y=E \backslash H(X) \text {. }
$$

Obviously, $H$ is a countable group and $Y \in I$. Further, it can easily be seen that every $H$-orbit contained in the set $E \backslash Y$ has a nonempty intersection with the given set $X$. From this fact it immediately follows that $X$ contains an $(H, I)$-selector. Thus, implication 1$) \Rightarrow 2)$ is proved.

The converse implication 2$) \Rightarrow 1$ ) is trivial since, for any countable subgroup $H$ of $G$, every $(H, I)$-selector is an $(H, I)$-thick subset of $E$.

Lemma 2 Let $(E, G)$ be a space equipped with a transformation group, $I$ be a $G$-invariant $\sigma$-ideal of subsets of $E$ and let $H$ be a countable subgroup of $G$. Then any two $(H, I)$-selectors are countably $(H, I)$-equidecomposable. 
This lemma is trivial.

We say that a set $X \subset E$ is $(\omega, G, I)$-divisible if there exists a disjoint family $\left\{X_{n}: n<\omega\right\}$ of subsets of $X$ such that

a) $X \backslash \cup\left\{X_{n}: n<\omega\right\} \in I$;

b) the sets $X_{n}(n<\omega)$ are pairwise countably $(G, I)$-equidecomposable.

Moreover, we say that a set $X \subset E$ is strongly $(\omega, G, I)$-divisible if there exists a disjoint family $\left\{X_{n}: n<\omega\right\}$ of subsets of $X$ satisfying relation a) and the following relation:

b') the sets $X_{n}(n<\omega)$ are pairwise $G$-congruent.

Lemma 3 Let $(E, G)$ be a space equipped with a transformation group, $I$ be a $G$-invariant $\sigma$-ideal of subsets of $E$ and let $H_{1}$ and $H_{2}$ be two countable subgroups of $G$ such that

1) $H_{1} \subset H_{2}$;

2) $\mathrm{H}_{2}$ acts I-freely in $\mathrm{E}$;

3) the index of $H_{1}$ in $H_{2}$ is equal to $\omega$.

Then there exists a strongly $(\omega, G, I)$-divisible $\left(H_{1}, I\right)$-selector in $E$.

Proof. Denote by $Z$ an arbitrary $H_{2}$-selector in $E$. Using this $H_{2}$-selector $Z$, we shall construct the desired $\left(H_{1}, I\right)$-selector. First of all we need a representation of the group $H_{2}$ in the form

$$
H_{2}=\cup\left\{H_{1} h_{n}: n<\omega\right\},
$$

where $\left\{h_{n}: n<\omega\right\}$ is a countable family of elements of $H_{2}$ such that

$$
H_{1} h_{n} \neq H_{1} h_{m}(n<\omega, m<\omega, n \neq m) .
$$

Further, we put

$$
X=\cup\left\{h_{n}(Z): n<\omega\right\} .
$$

Since $H_{2}$ acts $I$-freely in $E$, we have $g(Z) \cap h(Z) \in I$, for any two distinct transformations $g$ and $h$ from $H_{2}$. It immediately follows from this fact that the set $X$ is strongly $(\omega, G, I)$-divisible. Also, it can easily be checked that $X$ is an $\left(H_{1}, I\right)$-selector in $E$. Thus, $X$ is the required $\left(H_{1}, I\right)$-selector. 
Lemma 4 Let $(E, G)$ be a space equipped with a transformation group, I be a $G$-invariant $\sigma$-ideal of subsets of $E$ and let $H$ be a countable subgroup of $G$ acting $I$-freely in $E$. Suppose also that $X$ is an $(\omega, G, I)$-divisible $(H, I)$ selector in $E$. Then the sets $X$ and $E$ are countably $(G, I)$-equidecomposable.

Proof. According to our assumption, we can write

$$
X \backslash \cup\left\{X_{n}: n<\omega\right\} \in I,
$$

for some disjoint family $\left\{X_{n}: n<\omega\right\}$ consisting of sets $X_{n} \subset X$ which are pairwise countably $(G, I)$-equidecomposable. We have, on the other hand, that

$$
E \backslash \cup\{h(X): h \in H\} \in I
$$

and, consequently,

$$
E \backslash \cup\left\{h\left(X_{n}\right): h \in H, n<\omega\right\} \in I .
$$

Thus, we see that the basic set $E$ can be represented as the union (modulo $I$ ) of an infinite countable $I$-almost disjoint family of sets each of which is countably $G$-equidecomposable with the set $X_{0}$. Consequently, $X$ and $E$ are countably $(G, I)$-equidecomposable.

Now, we can formulate and prove the following statement.

Proposition 2 Let $(E, G)$ be a space equipped with a transformation group and let $I$ be a $G$-invariant $\sigma$-ideal of subsets of $E$. Suppose that the next two conditions are satisfied:

1) $\operatorname{card}(G)>\omega$

2) $G$ acts I-freely in $E$.

Let $X$ be a subset of $E$. Then the following assertions are equivalent:

a) $X$ is a $(G, I)$-thick set in $E$;

b) the sets $X$ and $E$ are countably $(G, I)$-equidecomposable.

Proof. Obviously, if $X$ is countably $(G, I)$-equidecomposable with $E$, then $X$ is a $(G, I)$-thick subset of $E$, i.e. implication $b) \Rightarrow a)$ is true for an arbitrary space $(E, G)$.

Let us establish implication $a) \Rightarrow b)$. Suppose that $X$ is $(G, I)$-thick in $E$, i.e. there exists a family $\left\{h_{n}: n<\omega\right\}$ of transformations from $G$ such that

$$
E \backslash \cup\left\{h_{n}(X): n<\omega\right\} \in I .
$$


Let us denote by $H$ the subgroup of $G$ generated by $\left\{h_{n}: n<\omega\right\}$. Since $H$ is countable and $G$ is uncountable, there exists a countable group $H^{\prime} \subset$ $G$ such that $H \subset H^{\prime}$ and the index of $H$ in $H^{\prime}$ is equal to $\omega$. Applying Lemma 3 to the groups $H$ and $H^{\prime}$, we obtain that there exists a strongly $(\omega, G, I)$-divisible $(H, I)$-selector $Z$. According to Lemma 4, the sets $Z$ and $E$ are countably $(G, I)$-equidecomposable. Further, according to Lemma 1, the given set $X$ contains a certain $(H, I)$-selector. This $(H, I)$-selector will be denoted by $Y$. Applying Lemma 2, we see that the sets $Y$ and $Z$ are countably $(G, I)$-equidecomposable. So, by transitivity, $Y$ and $E$ are countably $(G, I)$ equidecomposable, too. Finally, taking into account the relation $Y \subset X \subset E$, we conclude that $X$ is countably $(G, I)$-equidecomposable with $E$.

Remark 2. The referee suggested a shorter argument (skipping Lemmas 1 - 4) in order to give another proof of Proposition 2. However, we decided to preserve the above-mentioned Lemmas in the text, because they are not difficult and contain some additional information concerning $(G, I)$-thick sets and countable $(G, I)$-equidecomposability.

Example 4. An analogue of Proposition 2 is not true, in general, for a countable group $G$ of transformations of $E$. Moreover, suppose that $G$ is a group of transformations of $E$ such that there exists at least one point $x \in E$ for which we have the inequalities $1<\operatorname{card}(G(x)) \leq \omega$. Then it is not difficult to check that the set

$$
X=(E \backslash G(x)) \cup\{x\}
$$

is $G$-thick in $E$, but $X$ and $E$ are not countably $G$-equidecomposable.

Example 5. Starting with Proposition 2, one can easily obtain the existence of nonmeasurable sets with respect to a given probability invariant measure. More precisely, let $E$ be a basic set, $G$ be an uncountable group of transformations of $E$, and let $\mu$ be a complete probability $G$-invariant measure on $E$ such that $G$ acts $I(\mu)$-freely in $E$. Then we can assert that there exists a $\mu$-nonmeasurable subset of $E$. In order to show this, suppose to the contrary that $\operatorname{dom}(\mu)$ coincides with the family of all subsets of $E$. Let $H$ be an arbitrary subgroup of $G$ satisfying the inequalities

$$
1<\operatorname{card}(H) \leq \omega .
$$

Let us consider a set

$$
Z=\{z \in E: H(z)=\{z\}\} .
$$

Since $G$ acts $I(\mu)$-freely in $E$, we have $Z \in I(\mu)$. Obviously, for each point $z \in E \backslash Z$, the orbit $H(z)$ contains at least two distinct elements. Consequently, 
there are two disjoint $(H, I(\mu))$-selectors $X$ and $Y$. According to Proposition 2, each of the sets $X$ and $Y$ is countably $(G, I(\mu))$-equidecomposable with $E$. Therefore, we have the equalities

$$
\mu(X)=\mu(Y)=\mu(E)=1, \mu(X \cup Y)=2,
$$

which immediately imply a contradiction. This contradiction establishes the existence of subsets of $E$ nonmeasurable with respect to $\mu$.

It is reasonable to emphasize here that the argument presented above is essentially based on the assumption of invariance of $\mu$ and does not work for probability quasiinvariant measures.

The next example contains an application of Proposition 2 to the classical situation of a finite-dimensional Euclidean space equipped with a group of affine transformations.

Example 6. Let $E$ be the $n$-dimensional Euclidean space and let $G$ be an uncountable subgroup of the group of all affine transformations of $E$. We denote by $I$ the $\sigma$-ideal of subsets of $E$, generated by the family of all affine hyperplanes in $E$. Obviously, the $\sigma$-ideal $I$ is $G$-invariant, and $G$ acts $I$-freely in $E$. Thus, according to Proposition 2, a set $X \subset E$ is $(G, I)$-thick in $E$ if and only if the sets $E$ and $X$ are countably $(G, I)$-equidecomposable. The following question arises naturally: can the countable $(G, I)$-equidecomposability of sets $X$ and $E$ be strengthened to the usual countable $G$-equidecomposability of $X$ and $E$. Clearly, a necessary condition for this is the following one: there does not exist a nonempty $G$-invariant set belonging to $I$. This condition can also be formulated in another, geometrical, form: for each affine linear manifold $P \subset$ $E$ with $\operatorname{dim}(P)<n$, the family of manifolds $\{g(P): g \in G\}$ is uncountable. We are going to show that the latter condition is sufficient for deducing the countable $G$-equidecomposability of sets $X$ and $E$ from the countable $(G, I)$ equidecomposability of $X$ and $E$. So, in our further consideration, we assume that the group $G$ satisfies the above-mentioned condition.

First of all let us note that if $A$ and $B$ are any two subsets of $E$ and $A$ is countably $G$-equidecomposable with a subset of $B$, then we write $A \preceq_{G} B$. For various properties of the relation $\preceq_{G}$, see e.g. [18]. In particular, the conjunction of relations $A \preceq_{G} B$ and $B \preceq_{G} A$ is true if and only if the sets $A$ and $B$ are countably $G$-equidecomposable.

Let $Z$ be a set belonging to $I$. For a natural number $k<n$, we write $\operatorname{dim}(Z)=k$ if $Z$ can be represented as the union of a countable family of affine linear manifolds in $E$ such that each of those manifolds has dimension $k$. Let us prove, by induction on $k$, that the sets $E$ and $E \backslash Z$ are countably $G$-equidecomposable. This will give us the required result that the sets $X$ and 
$E$ are countably $(G, I)$-equidecomposable if and only if $X$ and $E$ are countably $G$-equidecomposable.

1. $\operatorname{dim}(Z)=0$. Evidently, in this case, $Z$ is a countable subset of $E$. Taking into account the fact that all $G$-orbits of points of $E$ are uncountable, it is not difficult to define a set $Y \subset E$ such that

a) $\operatorname{card}(Y)=\omega$ and $Z \subset Y$;

b) $Y \preceq_{G} Y \backslash Z$.

Therefore, we can write

$$
E=((E \backslash Y) \cup Y) \preceq_{G}((E \backslash Y) \cup(Y \backslash Z))=E \backslash Z,
$$

and, thus, the sets $E$ and $E \backslash Z$ are countably $G$-equidecomposable.

2. Suppose now that our assertion has already been established for all sets $Z \in I$ with $\operatorname{dim}(Z)<k$, where $k<n$, and let us prove that this assertion is also true for all those sets which belong to $I$ and have dimension $k$. Let us take any set $Z \in I$ with $\operatorname{dim}(Z)=k$. An argument similar to the one used in case 1 shows us that there exists a set $Y \in I$ such that

(a) $Z \subset Y$ and $\operatorname{dim}(Y)=k$;

(b) for some set $Z^{*} \subset Y$ with $\operatorname{dim}\left(Z^{*}\right)<k$, we have $Y \backslash Z^{*} \preceq_{G} Y \backslash Z$.

Therefore, we can write

$$
E \backslash Z^{*}=\left((E \backslash Y) \cup\left(Y \backslash Z^{*}\right)\right) \preceq_{G}((E \backslash Y) \cup(Y \backslash Z))=E \backslash Z .
$$

Applying the assumption of induction, we get

$$
E \preceq_{G} E \backslash Z^{*} \preceq_{G} E \backslash Z .
$$

Finally, since we also have $E \backslash Z \preceq{ }_{G} E$, we conclude that the sets $E$ and $E \backslash Z$ are countably $G$-equidecomposable.

In particular, we see that any two $(G, I)$-thick subsets of $E$ are countably $G$-equidecomposable. For instance, if $G$ acts transitively in $E$, then any two subsets of $E$ with nonempty interiors are countably $G$-equidecomposable (cf. [18] where an analogous statement is established only for the group of all translations of $E$ ).

Remark 3. A result similar to the one presented in Example 6 was also obtained in [12].

The next simple proposition yields a characterization of $(G, I)$-thin sets (and, consequently, of $(G, I)$-thick sets) in an arbitrary space $(E, G)$. 
Proposition 3 Let $(E, G)$ be a space equipped with a transformation group, $I$ be a $G$-invariant $\sigma$-ideal of subsets of $E$ and let $X$ be a subset of $E$. Then the following three assertions are equivalent:

1) $X$ is $(G, I)$-thin in $E$;

2) there exists a two-valued complete probability $G$-invariant measure $\mu$ on $E$ such that $I \subset I(\mu)$ and $X \in I(\mu)$;

3) there exists a nonzero $\sigma$-finite complete $G$-quasiinvariant measure $\mu$ on $E$ such that $I \subset I(\mu)$ and $X \in I(\mu)$.

Proof. Suppose that $X$ is $(G, I)$-thin in $E$. Then it is not hard to verify that there exists a proper $G$-invariant $\sigma$-ideal $J$ of subsets of $E$, containing $I \cup\{X\}$. Let us denote by $S$ the $\sigma$-algebra of subsets of $E$, generated by $J$. Obviously, $S$ is invariant under $G$ and, for each $Y \in S$, we have either $Y \in J$ or $E \backslash Y \in J$. Let us put $\mu(Y)=0$ if $Y \in J$, and $\mu(Y)=1$ if $E \backslash Y \in J$. Then $\mu$ is a two-valued complete probability $G$-invariant measure on $E$ for which $I(\mu)=J$. In particular, $I \subset I(\mu)$ and $X \in I(\mu)$. Thus, implication 1) $\Rightarrow 2)$ is proved.

The implications 2) $\Rightarrow 3$ ) and 3) $\Rightarrow 1$ ) are trivial.

We now return to the question concerning the existence of sets nonmeasurable with respect to general nonzero $\sigma$-finite quasiinvariant measures. More exactly, let $E$ be a basic set equipped with an uncountable group $G$ of its transformations and let $\mu$ be a nonzero $\sigma$-finite $G$-quasiinvariant measure on $E$ such that $G$ acts $I(\mu)$-freely in $E$. Does there exist a subset of $E$ nonmeasurable with respect to $\mu$ ? An affirmative answer to this question was obtained in the works [5], [7], and [13] (as mentioned in [5], the question was originally raised by Oxtoby). The method of these works can be regarded as a certain combination of the two classical approaches - an algebraic approach of Vitali [17] and a set-theoretical approach of Ulam [16]. Namely, the next two facts play an essential role for the method presented in [5], [7] and [13]:

a) if $G^{\prime}$ is a subgroup of $G$ with $\operatorname{card}\left(G^{\prime}\right)=\omega_{1}$ and $X$ is an arbitrary $\mu$-measurable $G^{\prime}$-selector in $E$, then $\mu(X)=0$;

b) $\omega_{1}$ is not a real-valued measurable cardinal, i.e. there does not exist a nonzero $\sigma$-finite diffused (continuous) measure defined on the family of all subsets of $\omega_{1}$.

We recall that statement b) was first established by Ulam in his famous work [16] where, in particular, he constructed a special $\left(\omega \times \omega_{1}\right)$-matrix consisting of subsets of $\omega_{1}$ (the construction of that matrix is also given in [11]). 
If we want to avoid the purely set-theoretical approach due to Ulam, we must investigate more thoroughly the algebraic structure of $G$-thin sets. Following this way, we can even get a stronger result. A tool for obtaining such a result is the notion of a $G$-negligible set, which is a particular case of the notion of a $G$-thin set.

Definition 6 We say that a $G$-thin subset $X$ of $E$ is $G$-negligible if, for each probability $G$-quasiinvariant measure $\mu$ on $E$ such that $G$ acts $I(\mu)$-freely in $E$, we have the implication $X \in \operatorname{dom}(\mu) \Rightarrow \mu(X)=0$.

Example 7. Let $\mathbb{R}^{2}$ denote the Euclidean plane and let $\Gamma$ be the group of all translations of $\mathbb{R}^{2}$. Let $Z$ be a subset of $\mathbb{R}^{2}$ such that there exists a function $f: \mathbb{R} \rightarrow \mathbb{R}$ whose graph coincides with $Z$. Then it is not difficult to show that $Z$ is a $\Gamma$-negligible subset of $\mathbb{R}^{2}$.

Various properties of $G$-negligible subsets of $E$ are considered in [8]. In particular, it is established there that, for $G$-negligible sets $X$ and $Y$, the set $X \cup Y$ need not be $G$-negligible.

Suppose now that $\operatorname{card}(G)=\omega_{1}$. Then, as proved in [8], there exists a partition $\left\{X_{k}: k<\omega\right\}$ of $E$ such that each set $X_{k}$ is $G$-negligible in $E$ (see also [9] where a more general result, in group-theoretical and topological terms, is presented). Actually, it was shown in [8] and [9] that each set $X_{k}$ of the above-mentioned partition is absolutely $G$-negligible with respect to the class of all those probability $G$-quasiinvariant measures $\mu$ on $E$ for which $G$ acts $I(\mu)$-freely in $E$ (the notion of an absolutely $G$-negligible subset of $E$ is a particular case of the notion of a $G$-negligible subset of $E$; it is defined and investigated in [8]).

We wish to emphasize that the latter result does not require the real-valued nonmeasurability of $\omega_{1}$ and immediately yields the next statement.

Proposition 4 Let $E$ be a set and let $G$ be an uncountable group of transformations of $E$. Then there exists a partition $\left\{X_{k}: k<\omega\right\}$ of $E$ with the following property: for every probability $G$-quasiinvariant measure $\mu$ on $E$ such that $G$ acts $I(\mu)$-freely in $E$, at least one of the sets $X_{k}$ is nonmeasurable with respect to $\mu$.

Proof. Let $G^{\prime}$ be a subgroup of $G$ satisfying the equality $\operatorname{card}\left(G^{\prime}\right)=\omega_{1}$. Then, as mentioned above, there exists a partition $\left\{X_{k}: k<\omega\right\}$ of $E$ consisting of $G^{\prime}$-negligible sets. Now, let $\mu$ be an arbitrary probability $G$ quasiinvariant measure on $E$ such that $G$ acts $I(\mu)$-freely in $E$. Obviously, $\mu$ is also a $G^{\prime}$-quasiinvariant measure and $G^{\prime}$ acts $I(\mu)$-freely in $E$. Suppose that all sets $X_{k}(k<\omega)$ are measurable with respect to $\mu$. Then we have 
$\mu\left(X_{k}\right)=0$, for all $k<\omega$. Consequently, $\mu(E)=0$, which contradicts the equality $\mu(E)=1$. This contradiction ends the proof.

We see, in particular, that if an uncountable group $G$ acts freely in $E$, then there exists a partition $\left\{X_{k}: k<\omega\right\}$ of $E$ with the following property: for every probability $G$-quasiinvariant measure $\mu$ on $E$, at least one of the sets $X_{k}$ is nonmeasurable with respect to $\mu$.

In connection with this result, let us remark that if $\mu$ is an arbitrary probability measure on $E$ and $\left\{Y_{k}: k<\omega\right\}$ is a countable partition of $E$, then there always exists a measure $\nu$ on $E$ extending $\mu$ and satisfying the relation

$$
\left\{Y_{k}: k<\omega\right\} \subset \operatorname{dom}(\nu) .
$$

For the proof, see [1] or [3]. Moreover, it can be proved that if $\mu$ is a probability measure on $E$ and $\left\{Y_{t}: t \in T\right\}$ is an arbitrary partition of $E$, then there exists a measure $\nu$ on $E$ extending $\mu$ and satisfying the relation

$$
\left\{Y_{t}: t \in T\right\} \subset \operatorname{dom}(\nu) .
$$

Thus, we see that the situation of quasiinvariant measures essentially differs from the situation of ordinary (i.e. nonquasiinvariant) measures.

Proposition 4 inspires us to formulate the following problem.

Problem. Let $G$ be an uncountable group of transformations of $E$, acting freely in $E$. Does there exist a finite partition of $E$ consisting of $G$-negligible sets?

More generally, it would be interesting to find a precise characterization of all those spaces $(E, G)$ for which such a partition does exist.

Example 8. Let $G$ be an arbitrary uncountable commutative group. Let us put $E=G$ and let us identify the group $G$ with the group of all translations of $E$. Hence, we have the space $(E, G)$ with a transformation group. It was shown in [10] that there exists a partition $\{A, B, C\}$ of $E$ such that each of the sets $A, B$ and $C$ is $G$-negligible in $E$. We thus see that, in the case of uncountable commutative groups, the answer to the question posed above is positive.

\section{References}

[1] A. Ascherl, J .Lehn, Two principles for extending probability measures, Manuscripta Math., 21 (1977), 43-50. 
[2] S. Banach, A. Tarski, Sur la decomposition des ensembles de points en parties respectivement congruentes, Fund. Math., 6 (1924), 244-277.

[3] D. Bierlein, Uber die Fortsetzung von Wahrscheinlichkeitsfeldern, Z. Wahr. Verw. Gebiete, 1 (1962), 28-46.

[4] Z. I. Borevich, I. R. Shafarevich, Number Theory, Izd. Nauka, Moskva (1985) (in Russian).

[5] P. Erdös, R. D. Mauldin, The nonexistence of certain invariant measures, Proc. Amer. Math. Soc., 59 (1976), 321-322.

[6] S. Kakutani, J. C. Oxtoby, Construction of a nonseparable invariant extension of the Lebesgue measure space, Ann. Math., 52 (1950), 580-590.

[7] A. B.Kharazishvili, Certain types of invariant measures, Dokl. Akad. Nauk SSSR, 222, no. 3 (1975), 538-540 (in Russian).

[8] A. B.Kharazishvili, Some Questions of Set Theory and Measure Theory, Izd. Tbil. Gos. Univ., Tbilisi (1978) (in Russian).

[9] A. B. Kharazishvili, Г-topology and the Baire property, Bull. Acad. Sci. of Georgia, 148, no. 1 (1993) (in Russian).

[10] A. Kharazishvili, Small sets in uncountable abelian groups, Acta Universitatis Lodziensis, Folia Mathematica, 7 (1995), 31-39.

[11] J. C. Oxtoby, Measure and Category, Springer-Verlag, Berlin (1971).

[12] M. Penconek, On nonparadoxical sets, Fund. Math., 139 (1991).

[13] C. Ryll-Nardzewski, R. Telgarsky, The nonexistence of universal invariant measures, Proc. Amer. Math. Soc., 69 (1978), 240-242.

[14] S. Solecki, On sets nonmeasurable with respect to invariant measures, Proc. Amer. Math. Soc., 119, no. 1 (1993), 115-124.

[15] S. Solecki, Measurability properties of sets of Vitali's type, Proc. Amer. Math. Soc., 119, no. 3 (1993), 897-902.

[16] S. Ulam, Zur Masstheorie in der allgemeinen Mengenlehre, Fund. Math., 16 (1930), 140-150.

[17] G. Vitali, Sul Problema della Misura dei Gruppi di Punti di una Retta, Bologna, Italy (1905).

[18] S. Wagon, The Banach-Tarski Paradox, Cambridge University Press, Cambridge (1985). 